\title{
Construction and evaluation of human papillomavirus genotype 18 pseudovirions
}

\author{
Sharifi $\mathbf{H}^{1,2}$, Barzegar $\mathbf{H}^{1,2}$, Langroudi $\mathbf{L}^{2}$, Azadmanesh $\mathbf{K}^{2}$, Arashkia $\mathbf{A}^{2^{*}}$ \\ ${ }^{1}$ Cellular and Molecular Biology Department, Pharmaceutical Sciences Branch, Islamic Azad University, Tehran, Iran. \\ ${ }^{2}$ Virology Department, Pasteur Institute of Iran, Tehran, Iran.
}

\begin{abstract}
Introduction: Cervical cancer is the second most common cancer in women worldwide and the role of human papillomavirus (HPV) has been proved in its etiology. The currentley available L1-capsid-protein-based vaccine is highly immunogenic and very high titers of serum antibodies can be obtained by its injection, but unfortunately it is restricted to only a few HPV genotypes and is relatively expensive. Therefore, development of the second-generation HPV vaccines has become the focus of the research and L2 capsid protein that is capable of producing broad spectrum antibodies has become one of the main candidates. Evaluation of the vaccine immunogenicity however, requires develoment of HPV pseudovirions (HPV PvSs) comprised of L1 and L2 virus protein and the present study was an attempt to produce PvSs of HPV18 genotype by an in-house method. Methods: The HPV18 L1/L2 coding plasmid and the reporter plasmid of pEGFP-N1 were amplified in E. coli DH5 $\alpha$ and purified using silica oxide method. The plasmids were co-transfected into HEK 293FT cell line and the preliminary analysis of expression was performed using fluorescence microscopy. The PvSs were partially purified using gel filtration chromatography and were used to transduce the 293FT cells to evaluate the infectivity rate of the PvSs. The results were analyzed by fluorescent microscopy, flow cytometry and atomic force microscopy. Results: The results showed that the HPV18 L1/L2 coding plasmid and the pEGFP-N1 reporter plasmid have been successfully co-transfected into HEK 293FT cells and the PvSs were constructed. The 293FT cells were successfully transduced by PvSs that had packaged reporter plasmid. These findings were confirmed by fluorescence microscopy and flow cytometry as well as AFM imaging. Conclusion: In this study, the cotransfection of HPV18 L1/L2 coding plasmid as well as pEGP-N1 reporter plasmid into the HEK 293FT cell led to the assembly of the pseudovirion harboring the reporter gene. The protocols used in this study were easy to perform and relatively inexpensive and did not rely on the commercial kits.
\end{abstract}

KEYWORDS: human papillomavirus, cervical cancer, pseudovirion.

\section{INTRODUCTION}

Human papillomavirus (HPV), a non-enveloped, double stranded-DNA virus is the most common cause of sexuallytransmitted infections with more than 100 known genotypes and genitally infecting HPVs have been divided into high- and low risk genotypes based on their ability to cause cancer [1]. There are at least 13 high risk oncogenic genotypes of which genotypes 16 and 18 have been strongly associated with cervical cancer. A yearly incidence of nearly 500,000 cases of cervical cancer worldwide has been estimated of which approximately $80 \%$ occur in developing countries, with a

*Corresponding Author: Arash Arashkia, Ph.D; Virology Department, Pasteur Institute of Iran, Tehran, Iran.

Email: a_arashkia@pasteur.ac.ir

Tel/Fax: (+98) 2166496682 mortality rate of nearly $50 \%$ [1-3]. Prevention through vaccinination has been the most effective measure in reducing the burden of the infectious diseases, but since the virus is difficult to culture, producing inactivated or attenuated virus as vaccine has not been possible [4]. Promising progress however has been made using spontaneously self assembled virus-like particles (VLP) obtained from purified L1 capsid protein of the virus which lack the viral genome and therefore are noninfectious, but exhibit conformational epitopes similar to the original HPV virions [5]. It has been shown that vaccination with VLPs induces a high titer of virus-neutralizing antibodies [6-8]. Currently two licensed VLP-based prophylactic vaccines are commercially available. The HPV16, HPV18 bivalent and HPVs 6, 11, 16, 18 tetravalent vaccines were developed by Merck and GlaxoSmithKline (GSK) respectively and are prepared from purified recombinant L1 proteins. Clinical 
evidence has shown the efficacy of these vaccines against the genotypes from which the L1 originates, but are ineffective against other high risk genotypes that are shown to be present at significant levels in many countries [9]. Efforts are underway to develop a nine-valent combination vaccine, but the inclusion of many VLP types might lead to antigenic competition resulting in lower protective efficacy while increasing the cost and complicating vaccine development [10]. To address these issues the use of L2 the minor capsid protein of the HPV has been proposed that in animal studies has been shown to induce broad-spectrum cross-neutralizing antibodies, thereby offering broader protection [11]. However it has been reported that the immune response to the low immunogenic L2 protein does not increase due to the immunodominance of L1 making incorporation of the L2 in L1 VLPs less effective necessiating the development of new vaccine strategies [12]. One such strategy is construction of HPV pseudovirions (PsV) consisting of the L1 and L2 virus proteins with the ability to attach and enter the cells [13]. Production of such particles requires the simultaneous expression of the L1 and L2 genes and their assembly on the surface of the PvSs. Furthermore, to evaluate the ability of the PvSs to enter the cells, a plasmid coding for a reporter gene is co-transfected with L1/L2 coding plasmid which would be packaged within the PvSs during the capsid assembly.

In this study construction and partial purification of HPV 18 PvSs, harboring EGFP reporter gene as a tool for assessment of neutralizing antibodies produced during vaccination was undertaken.

\section{MATERIALS and METHODS}

E. coli DH5 $\alpha$ and pEGFPN-1 plasmid (Clontech, USA) were from the stock in Virology Department of Pasteur Institute of Iran and the HEK 293FT cell line was purchased from Invitrogen (USA). The HPV18 L1- and L2-coding sequences cloned in an IRES-containing mammelian bicistronic expression vector which was kindly provided by DKFZ (Cancer Research Center, Germany) and named pHPV 18L1h/L2h. All restriction enzymes and molecular biology materials were purchased from Fermentas (Lithuania). Cell culture medium and supplements were obtained from GIBCO (USA).

\section{Amplification and purification of plasmids}

Separately transformed pEGFPN-1 and HPV18 L1h/L2h plasmids were maintained in E. coli $\mathrm{DH} 5 \alpha$ and purified for further manipulation using Triton-Silica method [14]. The purified plasmids were kept at $-20^{\circ} \mathrm{C}$ until used.

\section{HEK 293FT cell line transfection}

The HEK 293FT cells $\left(2 \times 10^{6}\right.$ cells $)$ were grown in DMEM medium supplemented with $10 \% \mathrm{FBS}, 100 \mathrm{IU} / \mathrm{ml}$ penicillin, and $100 \mu \mathrm{g} / \mathrm{ml}$ streptomycin (Gibco, USA) in $10 \mathrm{~cm}$ plates until the confluency of the cells reached $40-50 \%$. Calcium phosphate method was used for co-transfection of HEK 293FT cells with purified pEGFP-N1 and HPV18 L1h/L2h plasmids [15]. Briefley, $5 \mu \mathrm{g}$ of each palsmid in $438 \mu \mathrm{l}$ deionized sterile water was added by $62 \mu \mathrm{l}$ of $2 \mathrm{M} \mathrm{CaCl}_{2}$ and mixed with $500 \mu \mathrm{l}$ of $2 \mathrm{x}$ HBS buffer $\left(280 \mathrm{mM} \mathrm{NaCl}, 10 \mathrm{mM} \mathrm{KCl}, 1.5 \mathrm{mM} \mathrm{Na}_{2} \mathrm{HPO}_{4}, 12\right.$ $\mathrm{mM}$ Dextrose, and $50 \mathrm{mM}$ HEPES $\mathrm{pH}$ 7.05) and kept at room temperature for 10-15 minutes. The solution was then added drop wise to the HEK 293FT cells and incubated in $5 \% \mathrm{CO}_{2}$ at $37{ }^{\circ} \mathrm{C}$. The growth medium was replaced with fresh medium after $12 \mathrm{~h}$ and the cells were further incubated for $60 \mathrm{~h}$. The harvested cells were suspended in PBS buffer ( $\mathrm{pH}$ 7.4) and analyzed for EGFP expression by flow cytometery (CyFlow SL, Partec, Germany). About $2 \times 10^{6}$ cells were analyzed in the FL1 detector. Transfection efficiency was also determined visually by fluorescent microscopy. Visible light and $\mathrm{UV} /$ fluorescent images were captured at $10 \times$ magnification using an inverted microscope with fluorescence attachment (Jenus, China) and Panasonic Lumix DMC-LX5 compact digital camera (Panasonic, Japan). The digital images were level adjusted with Adobe Photoshop software to achieve an equivalent background, before the approximate percent of transfected cells was determined.

\section{Pseudovirion maturation and partial purification}

The HEK 293FT-transfected cells were trypsinized 60 h posttransfection, and were washed and pelleted by centrifugation. The cell pellet was washed twice and dissolved in 1.5 cell pellet volume of DPBS-Mg buffer (containing plain PBS added by 0.9 $\mathrm{mM} \mathrm{CaCl} 2,0.5 \mathrm{mM} \mathrm{MgCl}_{2}, 2.1 \mathrm{mM} \mathrm{KCl}$ and $350 \mathrm{mM} \mathrm{NaCl} \mathrm{pH}$ 7.4) and centrifuged at $200 \mathrm{x}$ g for $10 \mathrm{~min}$. The pellet was disolved in 1.5 cell pellet volume of DPBS-Mg buffer, and Triton X-100 $(0.5 \% \mathrm{v} / \mathrm{v})$ and RNase A (5 U/ $\mu \mathrm{l})$ were added to the suspension for cell lysis and ribosomal RNA removal. After $24 \mathrm{~h}$ incubation in $5 \% \mathrm{CO}_{2}$ at $37^{\circ} \mathrm{C}$ for pseudovirion maturation, the suspension was chilled on ice for $5 \mathrm{~min}$ and clarified by centrifugation at $5000 \mathrm{x}$ g for 10 minutes at $4^{\circ} \mathrm{C}$. The efficiency of the cell disruption was assessed by light microscopy.

For removal of Triton X-100 detergent, an in-house gel filteration chromatography method was used. Briefly, $1 \mathrm{ml}$ of Sephadex G-25 at the concentration of $30 \mathrm{~g} /$ liter with a ratio of Sephadex/PBS of $1: 1 \mathrm{v} / \mathrm{v}$ was poured in combed cotton-stuffed 1 ml-syringe. The syringe was placed on a drilled microcentrifuge tube, and then the system was entirely placed in a 50-ml conical centrifuge, and centrifuged $1 \mathrm{~min}$ at $4500 \times \mathrm{g}$. Then, $0.5 \mathrm{ml}$ of pseudovirus suspension was added to the syringe and centrifuged $1 \mathrm{~min}$ at $4500 \times \mathrm{g}$.

Ten microliters of the flow-through was diluted 1:100, 1:500 and 1:1000 and spotted on mica slides, air-dried and visualized by atomic force microscopy (NanoWizard II, JPK, Germany).

\section{Transduction of 293FT cells}

To evaluate the infectivity of the pseudovirions, the 293FT cells were grown in DMEM medium containing $10 \%$ FBS in 6-well plates (SPL, South Korea) in $5 \% \mathrm{CO}_{2}$ at $37^{\circ} \mathrm{C}$ until cell confluency was $40-50 \%$. The cells were trancduced by replacing the culture medium with fresh medium containing $1 / 50$ dilution of pseudovirions suspension flow-through from previous step and incubated in a $\mathrm{CO}_{2}$ incubator at $37^{\circ} \mathrm{C}$ for $48 \mathrm{~h}$. The efficiency of transduction of 293FT cells with the pseudovirions and expression of the reporter gene were evaluated by fluorescence microscopy and flow cytometry as described in the cell transfection methodology.

\section{RESULTS}

\section{Plasmid verification}

Restriction enzyme digestion of the HPV18 L1h/L2h and pEGFP-N1 plasmids extracted from E. coli DH5 $\alpha$, with HindIII resulted in $2100 \mathrm{bp}$ and $6600 \mathrm{bp}$ fragments for HPV18 $\mathrm{L} 1 \mathrm{~h} / \mathrm{L} 2 \mathrm{~h}$ and linearization of pEGFP-N1 respectively, verifying the authenticity of the plasmids (Fig. 1).

Transfection of HEK 293FT cells:

Expression of EGFP in HEK 293FT cells was observed after co-transfection of HPV18 L1h/L2h and pEGFP-N1 plasmids after $72 \mathrm{~h}$ (Fig. 2A). The calculated rate of transfection was 
approximately $80 \%$ which was determined by fluorescencent microscopy and confirmed by flow cytometry results (data not shown).

\section{Pseudovirions purification}

The HEK 293FT cells containing pseudovirions were sedimented and the cells were lysed, and the pseudoviral particles were partially purified by Sephadex G-25 before imaging by AFM (Fig. 2B). The size of pseudovirions was estimated as around $45-55 \mathrm{~nm}$.

\section{Transduction of 293FT cells}

The infectivity of the purified $\mathrm{PvSs}$ was evaluated by transducing the newly cultured HEK 293FT cells. Expression of the EGFP reporter gene in the cells indicated that pEGFP-N1 plasmid had been packaged inside the PvSs. The results showed that approximately $30 \%$ of transduced cells expressed the reporter gene as determined by flouresence microscopy (Fig. 2C) and flow cytometry (data not shown).

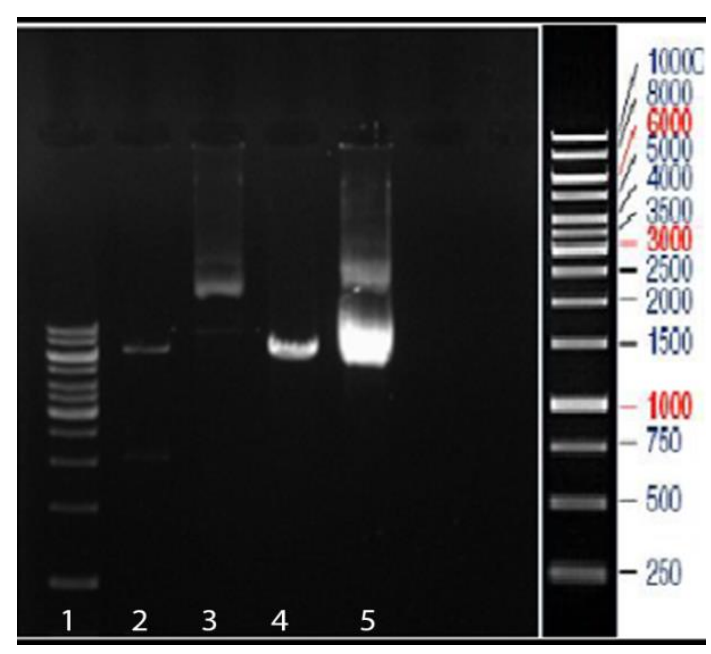

Fig. 1. Restriction enzyme digestion of pHPV18 L1h/L2h and pEGFP-N1 with HindIII, 1: 1 kb DNA weight marker, 2: digested pHPV18 L1h/L2h, 3: Un-digested pHPV18 L1h/L2h, 4: digested pEGFP-N1, 5: Un-digested pEGFP-N1.

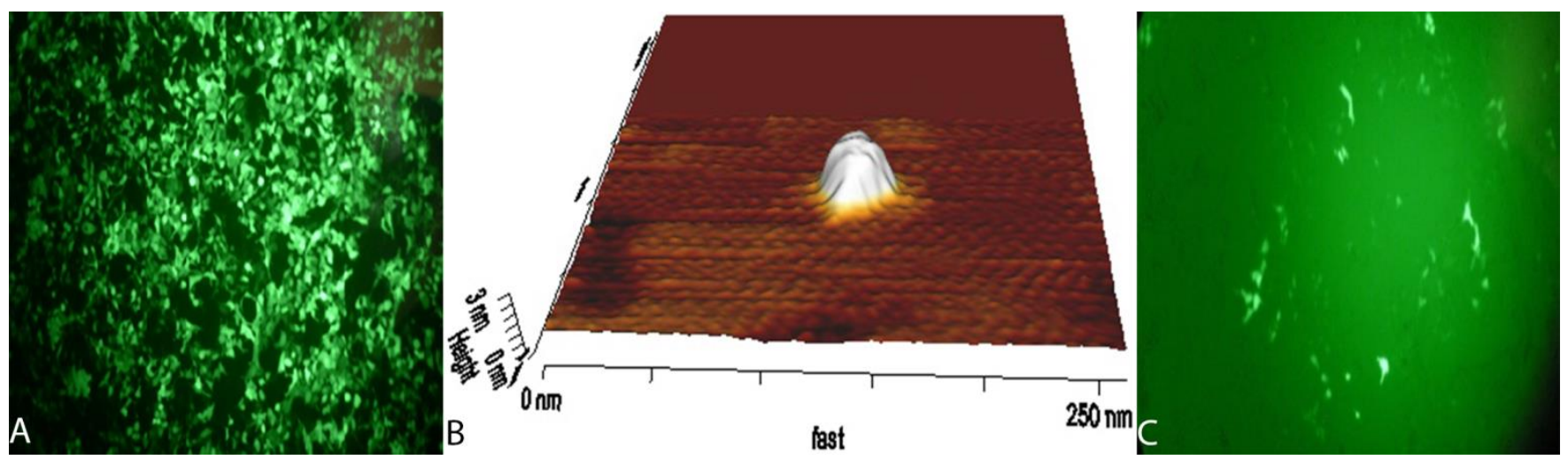

Fig. 2. Expression of EGFP in the transfected HEK 293FT cells (A), Atomic force microscopy image of pseudovirions extracted from HEK 293FT cells (B), HEK 293FT cells transduced by the HPV18 pseudovirions (C).

\section{DISCUSSION}

Cervical cancer is the second most common cancer among women and neutralizing antibodies are the most effective immune response to prevent the infection with HPV and the in vitro analysis of the magnitude of this response requires production of the HPV PsVs. Pseudovirions express the major and the minor L2 capsid proteins (L1 and L2), resemble the surface of the intact virus. PsVs can be used for vaccine and drug development and have become the standard tools for studies covering various aspects of the virus biology, for gene transfer or to determine the effectiveness of drug therapies [16, 17].

In this study, we prepared HPV18 PsVs with simple and less expensive methods that did not rely on commercial kits. Our data also showed that HPV PvSs prepared by these techniques are capable of infecting the HEK 293FT cell line in vitro.

Various methods and cell types have been used for production of PsVs, but it has been suggested that a single $75 \mathrm{~cm}^{2}$ flask of 293TT cells, a varient of HEK 293T cells engineered for high level expression of SV40 large and small T antigen required for amplification of the target plasmid containing SV40 origin of replication [18-19] is capable of producing a minimum of $1 \mathrm{mg}$ of transducing PsVs. However, the drawback to the use of this cell line despite its high yield is the posibility that the SV40 T antigen could be incorporated into the PsVs through promiscuous packaging turning the inocous PsVs into autonomous tumor viruses [19]. It has been reported that the yeild of PsVs in 293FT cell line is lower than that of 293TT cells due to either lower expression of SV40 T antigen in these cells or the lack of the SV40 small t antigen [19].

The transfection rate of $80 \%$ obtained in this study using calcium phosphate compared favorably with the more expensive commercial lipofectin. The reason for the low rate of infectivity (30\%) with PsVs was not ascertained, but the pseudovirions were only partially purified and the contaminating debries might have affected the infectivity rate. In this study we prepared HPV18 PsVs using an IREScontaining bicistronic vector coding for HPV18 L1 and L2 proteins that were packaged with a reporter plasmid that could be tracked to assess the infectivity of the pseudovirus particles. The methods employed were easy to perform and relatively inexpensive and did not rely on commercial kits which may facilitate their application in developing countries. 


\section{ACKNOWLEDGEMENT}

This research was supported by the grant no. 649 of Pasteur Institute of Iran.

\section{CONFLICT OF INTEREST}

The authors declare that they have no conflict of interest.

\section{REFERENCES}

1. Einstein MH. The immunological basis for immunization series: module 19: human papillomavirus infection. WHO; 2011, Geneva, Switzerland.

2. Cutts FT, Franceschi S, Goldie S, Castellsague X, de Sanjose S, Garnett $\mathrm{G}$ et al. Human papillomavirus and HPV vaccines: a review. Bull WHO 2016 .

3. Mahdavi A, Monk BJ. Vaccines against human papillomavirus and cervical cancer: promises and challenges. Oncologist. 2005;10:528-38.

4. Frazer HI. Development and implementation of papillomavirus prophylactic vaccines. J Immunol 2014;192:4007-11. doi: 10.4049/jimmunol.1490012.

5. Hernandez BY, Ton T, Shvetsov YB, Goodman MT, and Zhu X. Human papillomavirus (HPV) L1 and L1-L2 virus-like particle-based multiplex assays for measurement of HPV virion antibodies. CVI 2012;19:1348-52. doi:10.1128/CVI.00191-12.

6. Schiller JT, Nardelli-Haefliger D. Second generation HPV vaccines to prevent cervical cancer. Vaccine. 2006;31:S3/147-53.

7. Jagu S , Karanam B, Gambhira R ,Chivukula SV , Chaganti RJ , Lowy DR et al. Concatenated multitype L2 fusion proteins as candidate prophylactic pan-human papillomavirus vaccines. J Natl Cancer Inst. 2009;3(101):782-92.
8. Gambhira R, Karanam B, Jagu J, Roberts JN, Buck CB, Bossis I et al. A protective and broadly cross-neutralizing epitope of human papillomavirus L2. J Virol. 2007;81:13927-31.

9. Mariani L, Venuti A. HPV vaccine: an overview of immune response, clinical protection, and new approaches for the future. J Transl Med 2010;8:105. doi:10.1186/1479-5876-8-105.

10. Combelas N, Saussereau E, Fleury MJJ, RibeiroT, Gaitan J, Duarte-

Forero Df et al. Papillomavirus pseudovirions packaged with the L2 gene induce cross-neutralizing antibodies. J Transl Med 2010;8:28.

11. Gaukroger JM, Chandrachud LM, O’Neil BW, Grindlay GJ, Knowles G, Campo MS. Vaccination of cattle with bovine papillomavirus type 4 L2 elicits the production of virus-neutralizing antibodies. J Gen Virol 1996;77:1577-83.

12. Roden RB, Yutzy WH, Fallon R, Inglis S, Lowy DR, Schiller JT. Minor capsid protein of human genital papillomaviruses contains subdominant, crossneutralizing epitopes. Virology. 2000;270:254-7.

13. Lowy DR, Schiller JT. Prophylactic human papillomavirus vaccines. J Clin Invest. 2006;116:1167-73.

14. Grimm S, Voss-Neudecker F. High-purity plasmid isolation using silica oxide. Methods Mol Biol. 2003;235:83-7.

15. Robert E, Claudia A. Chen, John K. Rose. Calcium Phosphate Transfection. Curr Protoc Mol Biol. doi: 10.1002/0471142727.mb0901s63

16. Kumar S, Biswas M, Jose T. HPV vaccine: Current status and future directions. Med J Armed Forces India. 2015;71:171-7.

17. Gordon SN, Kines RC, Kutsyna G, Ma Z, Hryniewicz A, Roberts JN et al. Targeting the vaginal mucosa with human papillomavirus pseudovirion vaccines delivering Simian immunodeficiency virus DNA. J Immunol. 2012;188:714-23.

18. Peng S, Monie A, Kang TH, Hung C-F, Roden R and Wu T-C. Efficient delivery of DNA vaccines using human papillomavirus pseudovirions. Gene Ther 2010;17:1453-64.

19. Buck CB, Pastrana DV, Lowy DR, and Schiller JT. Generation of HPV pseudovirions using transfection and their use in neutralization assays. Meth Mol Med 2005, Vol. 119: Human Papillomaviruses: Methods and Protocols. Ed Davy C and Doorbar J. C Humana Press Inc., Totowa, NJ. 